\title{
Dual channel speech enhancement using particle swarm optimization
}

\author{
Dalal Hamza, Tariq Tashan \\ Electrical Engineering Department, College of Engineering, Mustansiriyah University, Iraq
}

\begin{tabular}{l} 
Article Info \\
\hline Article history: \\
Received Jan 14, 2021 \\
Revised Jun 30, 2021 \\
Accepted Jul 7, 2021 \\
\hline
\end{tabular}

\section{Keywords:}

Adaptive filter

Noise cancellation

Particle swarm optimization

Speech enhancement

\begin{abstract}
Adaptive processing for canceling noise is a powerful technology for signal processing that can completely remove background noise. In general, various adaptive filter algorithms are used, many of which can lack the stability to handle the convergence rate, the number of filter coefficient variations, and error accuracy within tolerances. Unlike traditional methods, to accomplish these desirable characteristics as well as to efficiently cancel noise, in this paper, the cancelation of noise is formulated as a problem of coefficient optimization, where the particle swarm optimization (PSO) is employed. The PSO is structured to minimize the error by using a very short segment of the corrupted speech. In contrast to the recent and conventional adaptive noise cancellation methods, the simulation results indicate that the proposed algorithm has better capability of noise cancelation. The results show great improvement in signal to noise ratio (SNR) of $96.07 \mathrm{~dB}$ and $124.54 \mathrm{~dB}$ for finite impulse response (FIR) and infinite impulse response (IIR) adaptive filters respectively.
\end{abstract}

This is an open access article under the CC BY-SA license.

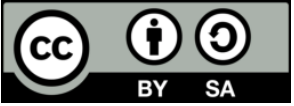

\section{Corresponding Author:}

Tariq Tashan

Electrical Engineering Department

College of Engineering

Mustansiriyah University, Baghdad, Iraq

Email: tariq.tashan@uomustansiriyah.edu.iq

\section{INTRODUCTION}

An adaptive noise canceller is a powerful tool that separates the desired speech signal from the background noise, which resulting in higher signal to noise ratio (SNR) of the desired speech signal. The adaptive filter has the capability to change its parameters based on specific condition, to handle the unknown statistics of the input signal. Many methods are used to optimize the adaptive filter coefficients. Several speech enhancing algorithms have been suggested [1], [2]. The least mean square (LMS), normalized LMS (NLMS), and genetic algorithm (GA) [3], [4] are adaptive filtering methods used for speech improvement. One of the popular algorithms used in this type of application is the LMS [5]. In terms of SNR enhancement, the NLMS outperforms the LMS algorithm. The NLMS features different step sizes, which allows it to converge faster [6]. For speech enhancement, other adaptive algorithms such as recursive least square (RLS) also used [7]. Kumar [8] presents the RLS algorithm to produce very low mean square error (MSE) and fast convergence, but having more computational complexity when compared to the LMS algorithm. So for different noise canceling applications, LMS is recommended over RLS methods, because RLS has more computational cost and stability issues than LMS-based methods. In [6] LMS has been developed in a variety of forms, the experimenal results show that the LMS algorithm is relatively better in terms of simplicity and application, when compared to LMS-based algorithms such as leaky LMS, block LMS, sign-data LMS, modified normalized LMS algorithm, sign-sign LMS algorithm, sign-sign LMS algorith with leakage term, 
sign-data normalized LMS, variable step-size LMS algorithm, filtered X-LMS algorithm, filtered X-LMS algorithm, frequency response shaped LMS algorithm, and hybrid LMS algorithm.

Global optimum solutions in multimodal error surface, are not easy to find when gradient-based algorithm is used. Therefore, stochastic optimization algorithms are used as alternative to the gradient descent-based techniques. Asl and Geravanchizadeha [9] present a new algorithm, based on sexual reproduction-based PSO (SRPSO) claiming enhanced signal of $14.37 \mathrm{~dB}$ [9]. The PSO, proposed by Kennedy and Eberhart [10] in 1995, as a class of swarm intelligence techniques, the PSO is the most common algorithm in the class of swarm intelligence methods [10], [11]. The benefit is that the algorithm is simple, requires fewer tuning parameters, and requires less information of gradient. In addition, it convergences faster, and it is better in finding global optimum solutions. Currently, the PSO is used to optimize the fitness function, in many problems like pattern classification, neural networks, and fuzzy control systems [12].

In the last decade, several methods are proposed for speech enhancement applications. A hybrid optimization algorithm, which employs the combination of the conventional $\theta$-PSO, and the shuffled subswarms particle optimization (SSPSO) technique. The hybrid algorithm is called $\theta$-SSPSO, achieving global convergence, for adaptive filters and SNR-improvement of $23.7804 \mathrm{~dB}$ [13]. An average SNR of 25.043 dB is claimed by Jose and Anoop [14], using a cuckoo search based algorithm. Kunche et al. [15] suggest an accelerated PSO (APSO) technique, with a comparison with the standard PSO, an SNR of $25.25 \mathrm{~dB}$ is claimed. Mahbub et al. [16] suggest a frequency domain based PSO, in terms of SNR enhancement, demonstrate superior results under different acoustic conditions. Asl and Nezhad [17], [18] present a modified PSO for speech enhancement, claiming improvement in SNR from $20.47 \mathrm{~dB}$ to $26.59 \mathrm{~dB}$. PSO is most suitable among other like LMS, NLMS, RLS as stated in [19], it is also outperforms other evolutionary algorithms such as bacteria foraging optimization (BFO), GA and artificial bee colony (ABC) as suggested in [20], [21]. Kunche et al. [22] claimed improvement in SNR about $24 \mathrm{~dB}$, when using a hybrid of PSO and gravitational search algorithm (GSA), which referred as PSOGSA, claiming better background noise reduction of the noisy input speech. However, it adds more complexity. An SNR of $32.97 \mathrm{~dB}$ is claimed by Selvi and Suresh [23] when proposing a hybrid technique, for speech enhancement, by using minimum MSE (MMSE) and PSO. Ramil et al. [24] a comparison of the performance of two systems, adaptive noise cancellation and adaptive line enhancer, is presented using objective and subjective measurements, for speech intelligibility, such as using the mean opinion score, MSE, and SNR, with selectable algorithms for the systems such as the NLMS, affine projection, dynamic set-membership affine projection. The experimental results show that the SNR by single input adaptive noise cancellation is (about $65.281 \mathrm{~dB}$ ) better than dual input adaptive noise cancellation (about $45.522 \mathrm{~dB}$ ). Bolisetty and Santiprabha [25] A novel approach for speech enhancement using modified wiener filtering is developed, and power spectrum computation and MMSE technique are applied, to overcome communications speech signals issues, and compared to other techniques which description in [26], the paper considers the NOIZEUS database. The results claim that the suggested model provides better SNR than other techniques for airport, car, and train noise with SNR values of $4.36 \mathrm{~dB}, 4.77 \mathrm{~dB}$, and $4.47 \mathrm{~dB}$ respectively. Kunche et al. [27] present a comparative study, by using the NOIZEUS and NOISEX datasets, to evaluate speech enhancement algorithms, like the bat algorithm (BA), standard PSO, APSO, GSA, and the hybrid PSOGSA. An improvement in SNR of $31 \mathrm{~dB}$ is claimed when BA is used. The PSO algorithm receives wide attention, due to its important advantages, in the process of speech enhancement [28], it successfully repairs the signal with reduced noise, especially in high frequency voice regions [29].

In this paper, the PSO algorithm is proposed for dual-channel speech enhancement. Unlike traditional iterative algorithms, that requires instantaneous access to the distorted speech signal, the proposed algorithm uses a very small portion of the distorted signal, which improves both local and global search. The rest of the paper is organized as follows: section 2 describes the dual-channel adaptive filter and its importance in enhancement speech, section 3 explains the PSO algorithm, section 4 illustrates the proposed algorithm, section 5 addresses the simulation results, while section 6 presents the main conclusions.

\section{ADAPTIVE FILTER FOR SPEECH ENHANCEMENT}

The Adaptive filter method is one of the important speech enhancement methods. A system with a linear filter is an adaptive filter, such filter has a transfer function of tunable parameters. These parameters can be tuned using an optimization method. The adaptive filter produces a clean signal, free from any undesired noise and other interfering signals. In an adaptive part, the filter properties are controlled, to reach the required values by interacting with the environment model, unlike traditional filter design techniques, that rely on fixed filter parameters, and no prior information known.

The block diagram of the dual-channel enhancement speech system is shown in Figure 1, where s[k] refers to the original clean speech signal, which occurs in one channel, $\mathrm{y}[\mathrm{k}]$ refers to the corrupted signal 
after adding the background noise $\mathrm{n}[\mathrm{k}]$. The reference noise signal $\mathrm{x}[\mathrm{k}]$ in the second channel, is correlated to the background noise $n[k]$. The filter output $n e[k]$ represents an estimation of $n[k]$. The expression for filter output, the MSE, and the error respectively are given by:

$$
\begin{aligned}
& n_{e}[k]=\sum_{n=0}^{N-1}(\mathrm{w}[\mathrm{n}] * \mathrm{x}[\mathrm{k}-\mathrm{n}]) \\
& M S E=\frac{1}{\mathrm{~L}} \sum_{\mathrm{n}=1}^{\mathrm{L}}[(\mathrm{e}[n]-\mathrm{s}[\mathrm{n}])]^{2} \\
& e[k]=\mathrm{y}[\mathrm{k}]-n_{e}[k]
\end{aligned}
$$

Where $\mathrm{N}$ is the finite impulse response (FIR) filter order, $\mathrm{w}[\mathrm{n}]$ is the $\mathrm{nth}$ coefficient, and $\mathrm{L}$ is the length of a speech signal. Adaptive filters contain two different units:

The first is the digital filter, which has tuneable coefficients, that can be adjusted to minimize the error. This filter can be modeled as FIR or infinite impulse response (IIR). Most nonlinear and iterative systems are represented as IIR systems. In this paper both FIR and IIR based adaptive filters are investigated, to represent the filter model between the two channels of the dual-channel optimization system.

The second unite is the adaptive algorithm, which updates the filter parameters, it must guarantee the fastest possible convergence to the optimum parameters, to meet the target value of the error. Many adaptive algorithms apply improvements, to traditional iterative procedures, to handle the function minimization problem.

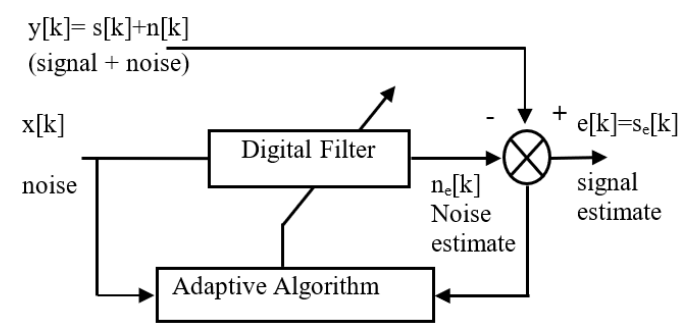

Figure 1. Effects Speech dual-channel model

\section{PARTICLE SWARM OPTIMIZATION (PSO)}

The PSO is an algorithm for adaptive noise cancellation, that is based on stochastic global optimization techniques [30]. Motivated by bird flocking and fish swarms' social engagement animals, this algorithm is proposed by Kennedy and Eberhart in 1995 [10]. The goal of PSO is to minimize residual noise by defining optimal adaptive filter weight coefficients. The size of the swarm or number of particles, number of iterations, velocity components, and acceleration coefficients are the basic PSO parameters. The best position of the whole swarm represents the best global solution and called $\mathrm{G}_{\mathrm{Best}}$, while the best solution, that

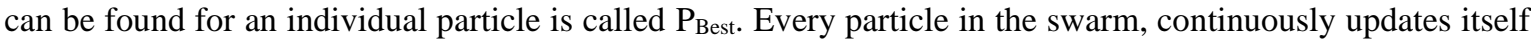
by taking in consideration both $G_{\text {Best }}$ and $P_{\text {Best. }}$ A new generation of particles is then formed. The fitness function, which is designed according to the optimization problem, evaluates the degree, to which the swarm is good or poor in functional operations. It is possible to consider each particle in the PSO as a point in the solution space. If the swarm size is $\mathrm{M}$, then the location of the $\mathrm{i}^{\text {th }}(\mathrm{i}=1,2, \ldots, \mathrm{M}) \mathrm{P}_{\mathrm{i}}$ is represented as a particle. The total swarm particles form a set $\mathrm{S}$. $\mathrm{P}_{\text {Besti }}$ is expressed as the "best" position, that can be reached by the particle. $\mathrm{V}_{\mathrm{i}}$ refers to the velocity of the $\mathrm{i}^{\text {th }}$ particle. The best particle among the swarm at each iteration is referred as $G_{\text {Best. }}$. Therefore, according to the following equation each particle $P_{i}$ updates its speed and position:

$$
\begin{aligned}
& V_{i, t+1}=W V_{t}+C_{1} r_{1}\left(P_{B e s t}-P_{i, t}\right)-C_{2} r_{2}\left(G_{B e s t, t}-P_{i, t}\right) \\
& P_{i, t+1}=P_{i, t}+V_{i, t+1}
\end{aligned}
$$

where $\mathrm{W}$ is the inertia weight, the cognition learning factor is $C_{l}$ and the social learning factor is $C_{2}$. The $r_{1}$ and $r_{2}$ are two random parameters, distributed uniformly in the interval $[0,1]$. P $P_{\text {Best }}$ represents the best solution, thus far achieved by individual particle, while $\mathrm{G}_{\mathrm{Best}}$ is the best value which so far obtained by all particles, $t$ represents the iterations index. Figure 2 shows the main stages of the PSO algorithm. 


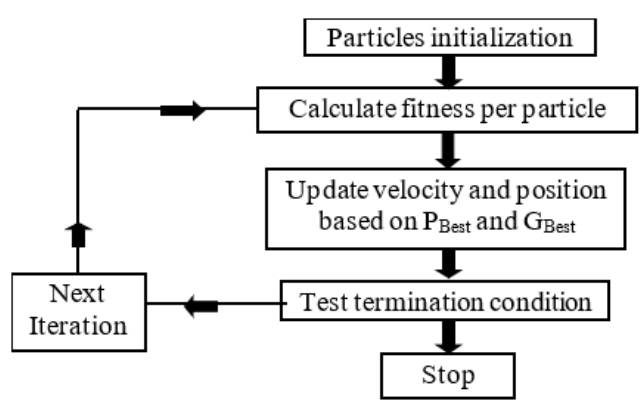

Figure 2. The PSO algorithm's flowchart

\section{PROPOSED ALGORITHM}

The proposed algorithm block diagram is shown in Figure 3 Different adaptive algorithms are suggested in the literature, such as LMS, PSO, and other optimization methods [20]. In all these methods, the adaptive algorithm works on minimizing the energy of the output signal $\mathrm{e}[\mathrm{k}]$, taking in consideration the whole speech signal. However, the task of the adaptive filter is to mimic the transfer function F[z], which represents the relation between the noise source $\mathrm{x}[\mathrm{k}]$ and the added noise $\mathrm{n}[\mathrm{k}]$. The estimation of such system is not necessarily related to the length of the speech signal. Therefore, in this paper a very small segment of the input signal (about $12.5 \mathrm{~ms}$ ) is considered, to optimize the adaptive filter coefficients. This short segment is preferred to be a nonspeech segment (i.e. only $\mathrm{n}[\mathrm{k}]$ ), so that the optimization algorithm can work effectively, since its task is to minimize the energy at the output to zero value, and consequently finding the optimum coefficients value. By considering such short segment of the audio input signal, the PSO algorithm can reach the optimum solution substantially faster than other traditional methods, which are limited by the instantaneous and/or the whole audio signal. The only limitation is if the transfer function $\mathrm{F}[\mathrm{z}]$ may change over time, which is not the case here, where $\mathrm{F}[\mathrm{z}]$ considered to be stationary.

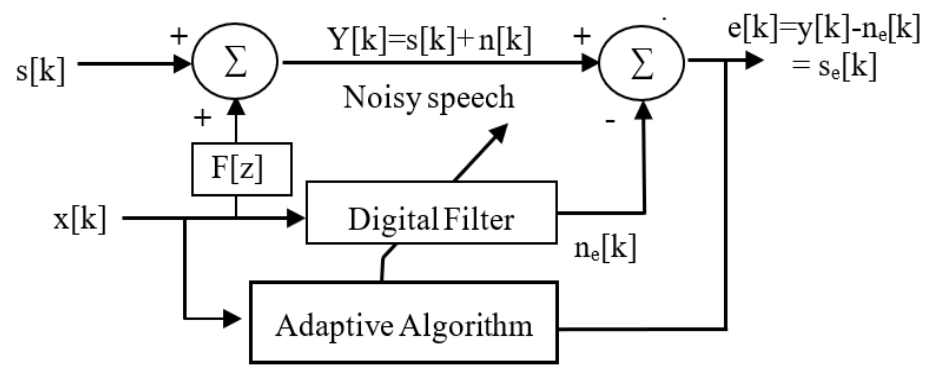

Figure 3. Adaptive filter for noise cancellation

\section{RESULTS}

In the experimental analysis, The PSO algorithm is implemented in MATLAB R2018b using a laptop of Core i7 processor, 8 GB RAM, mechanical hard disk. The reference noise in the second channel is filtered using the PSO and the LMS algorithms, to compare the efficiency of both algorithms. The performance index is the SNR and defined as (4).

$$
S N R=10 \log \left(\frac{\sum s^{2}[k]}{\sum(s[k]-y[k])^{2}}\right)
$$

The experiments are carried out using the NOIZEUS database [31] and the NOISEX-92 database. Eight noise types are selected from the NOISEX-92 [32] database (airport, babble, exhibition, car, restaurant, street, train, and subway). Clean speech samples are taken from the NOIZEUS dataset. Both clean speech and noise samples are recorded at the sampling frequency of $8 \mathrm{kHz}$. To obtain the corrupted speech signal, the reference noise is passed through the transfer function $\mathrm{F}[\mathrm{z}]$, which represents the recording environment model. Both FIR and IIR filter models are investigated for the $F[z]$. The filter $F[z]$ provided in (5) is considered in the simulation [13]. 
$F[z]=\frac{1}{1-1.2 \mathrm{Z}^{-1}+0.36 \mathrm{Z}^{-2}}$

The $\mathrm{W}[\mathrm{z}]$ adaptive filter is given by:

$$
\begin{aligned}
& W[z]=\frac{1}{1+P_{2}^{i} Z^{-1}+P_{1}^{i} Z^{-2}} \\
& F[z]=\sum_{k=0}^{M} b[k] Z^{-k}=-1.2 Z^{-1}+0.36 Z^{-2} \\
& W[z]=P_{2}^{i} Z^{-1}+P_{1}^{i} Z^{-2}
\end{aligned}
$$

The weights of the W[z] adaptive filter are calculated using the PSO. Table 1 illustrates the experimental conditions of the PSO algorithm for a short segment taken from a corrupted signal. Table 2 and Table 3 address a comparison of PSO and LMS algorithms based on SNR improvement by considering FIR and IIR models for F[z] respectively. Table 4 shows a comparison of SNR values obtained from different methods in the literature, at noise levels of $-5 \mathrm{~dB}$ and $-10 \mathrm{~dB}$, compared with the proposed algorithm based IIR adaptive filter. The proposed algorithm significantly outperforms the other methods, due to the accurate estimation of the F[z] model in the adaptive filter. Figure 4 shows a waveform example of the sample from the database, and the enhanced signal using PSO and LMS algorithms.

Table 1. PSO parameters

\begin{tabular}{ll}
\hline \multicolumn{1}{c}{ Parameter } & Value \\
\hline $\mathrm{C}_{1}$ & 1 \\
$\mathrm{C}_{2}$ & 2 \\
Iteration size & 100 \\
$\mathrm{~W}$ & 0.8 \\
adaptive filter taps number & 2 \\
Swarm size & 100 \\
\hline
\end{tabular}

Table 2. SNR-improvement at different noise levels

\begin{tabular}{ccc}
\multicolumn{3}{c}{ for FIR model of F[z] } \\
\hline $\begin{array}{c}\text { SNR noisy signal } \\
(\mathrm{dB})\end{array}$ & Algorithm & $\begin{array}{c}\text { SNR enhanced signal } \\
(\mathrm{dB})\end{array}$ \\
\hline-5 & PSO & 79.01 \\
& LMS & 7.08 \\
-12 & PSO & 85.77 \\
& LMS & 12.09 \\
-24 & PSO & 96.07 \\
& LMS & 23.55 \\
\hline
\end{tabular}

Table 3. SNR-improvement at different noise levels

\begin{tabular}{ccc}
\multicolumn{3}{c}{ for IIR model of F[z] } \\
\hline $\begin{array}{c}\text { SNR noisy signal } \\
(\mathrm{dB})\end{array}$ & Algorithm & $\begin{array}{c}\text { SNR enhanced signal } \\
(\mathrm{dB})\end{array}$ \\
\hline-5 & PSO & 122.9 \\
& LMS & 7.08 \\
-12 & PSO & 124.54 \\
& LMS & 12.09 \\
-24 & PSO & 114.88 \\
& LMS & 23.55 \\
\hline
\end{tabular}

Table 4. SNR comparison of different methods

\begin{tabular}{cll}
\hline SNR noisy signal (dB) & Algorithm & SNR enhanced signal (dB) \\
\hline \multirow{4}{*}{-5} & Proposed PSO & 122.9 \\
& LMS & 7.08 \\
& PSOGSA [22] & 24 \\
& GSA [22] & 22 \\
& Proposed PSO & 232.7729 \\
& A-SSPSO [13] & 23.7804 \\
& BA [27] & 31 \\
& BFO [20] & 0.1109 \\
-10 & GA [20] & 0.3141 \\
& ABC [20] & 0.3095 \\
& RLS [20] & -0.259 \\
& APSO [15] & 25.25 \\
& GSA [27] & 27 \\
\hline
\end{tabular}

Thirty clean speech samples are considered from the NOIZEUS database, each sample is corrupted with eight types of noise from the NOISEX-92 database. The SNR of the 30 samples corrupted with "airport" noise is shown in the Figure 5. The SNR enhancement of one sample from the NOIZEUS database, which corrupted with eight types of noise, using the FIR model in Figure 6 and the IIR model in Figure 7. 


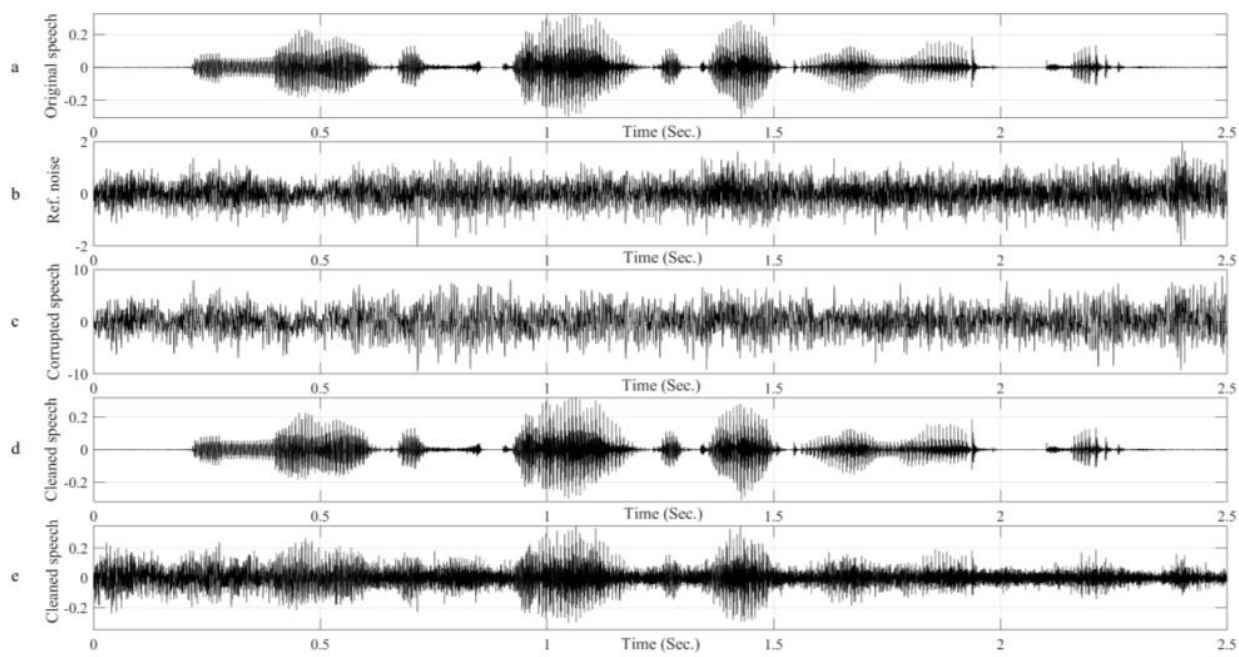

Figure 4. Enhancement of a standard waveform distorted by airport noise at -24dB SNR. (a) Original signal,

(b) Noise signal, (c) corrupted signal, (d) cleaned signal using PSO, and (e) cleaned signal using LMS

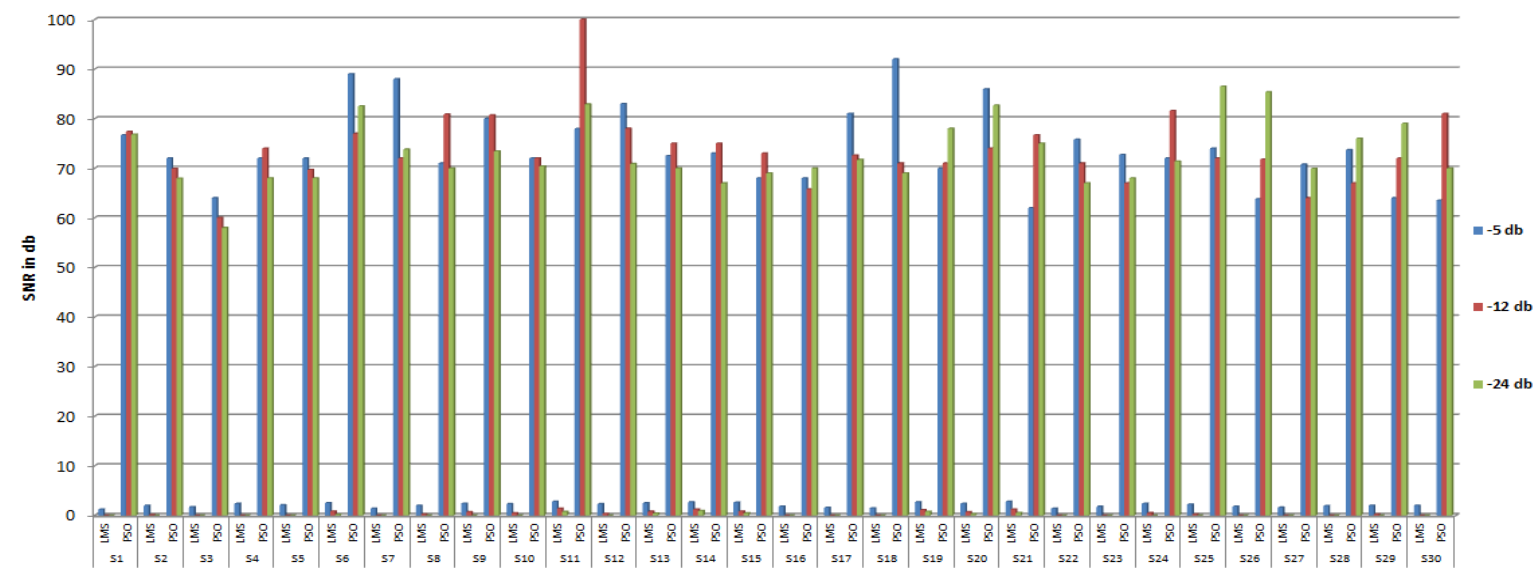

Figure 5. The performance of the proposed algorithm and LMS algorithm for 30 samples at different noise levels for FIR model

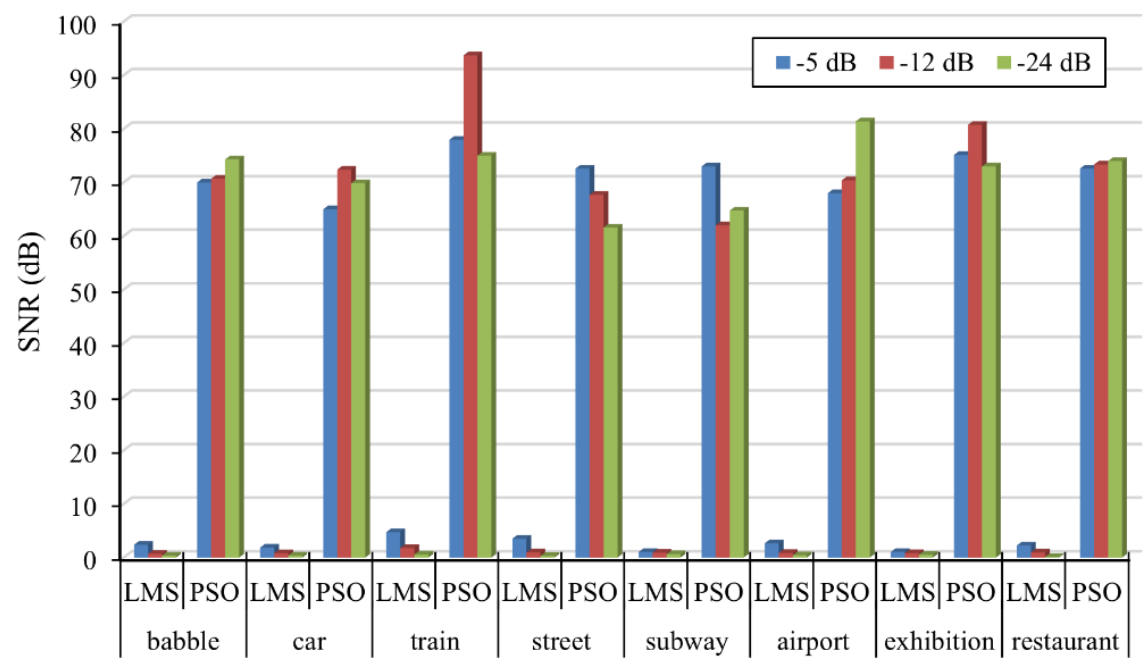

Figure 6. Enhancement of SNR of one sample corrupted with eight types of noise for FIR model at different noise levels 


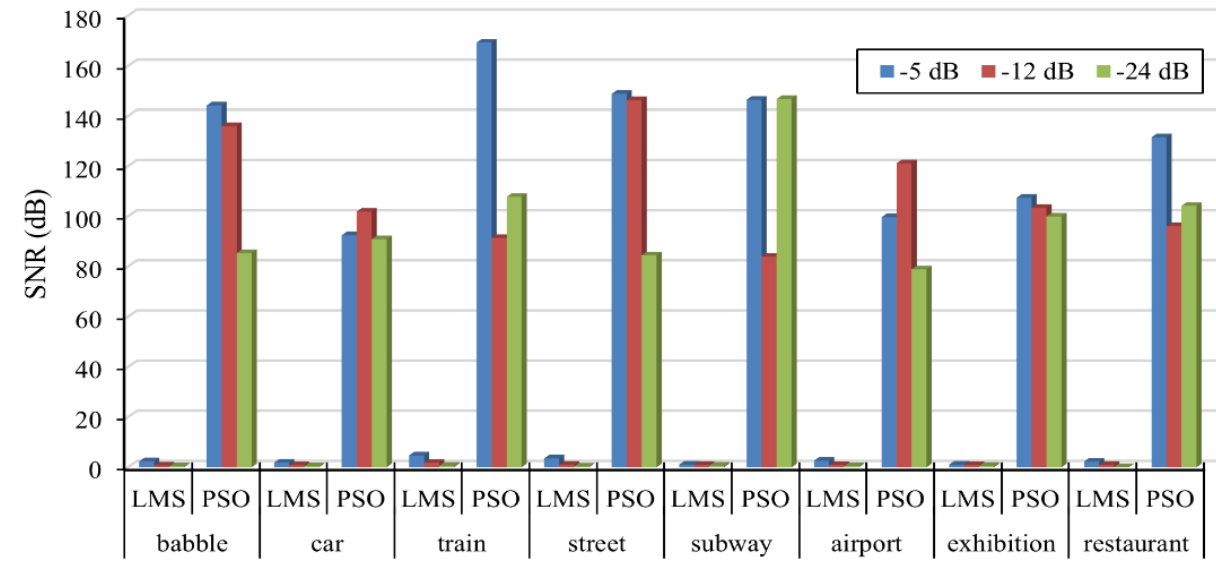

Figure 7. Enhancement of SNR of one sample corrupted with eight types of noise for IIR model at different noise levels

\section{CONCLUSION}

In this paper, an efficient and simple PSO-based adaptive noise cancelation algorithm is presented, to handle the problem of speech signal enhancement in a dual-channel model. The adaptive filter can estimate the recording environment model $\mathrm{F}[\mathrm{z}]$, by minimizing the mean square error, between the outputs of the two channels. The proposed algorithm uses only a short segment of the noisy audio file (about $12.5 \mathrm{~ms}$ ), to estimate the $\mathrm{F}[\mathrm{z}]$ model, once the model is correctly estimated, the adaptive filter can efficiently clean the rest of the noisy signal. The results are compared with the LMS algorithm and other methods in the literature. The LMS algorithm suffers from the difficulty, to find the desired solution in the search space, and it has little accuracy. The main advantage of using the proposed algorithm is the use of a short segment of the speech signal to estimate the $\mathrm{F}[\mathrm{z}]$ coefficients, this reduces the processing time of the PSO substantially, which makes the proposed algorithm plausible to be implemented in real time speech enhancement. Since, a short speech signal is considered in the PSO algorithm, it converges faster to the optimum solution. The results show significant superiority for the proposed algorithm over other methods as addressed in Table 4 .

\section{ACKNOWLEDGEMENTS}

The Authors would like to thank all colleagues at Electrical Engineering Department, College of Engineering, Mustansiriyah University for their support.

\section{REFERENCES}

[1] C. Plapous, C. Marro, L. Mauuary, and P. Scalart, "A two-step noise reduction technique," 2004 IEEE International Conference on Acoustics, Speech, and Signal Processing, 2004, pp. I-289, doi: 10.1109/ICASSP.2004.1325979.

[2] B. Kumar, "Comparative performance evaluation of MMSE-based speech enhancement techniques through simulation and real-time implementation," International Journal of Speech Technology, vol. 21, no. 4, pp. 1033-1044, 2018, doi: 10.1007/s10772-018-09567-5.

[3] P. Gupta, M. Patidar, and P. Nema, "Performance analysis of speech enhancement using LMS, NLMS and UNANR algorithms," 2015 International Conference on Computer, Communication and Control (IC4), 2015, pp. 1-5, doi: 10.1109/IC4.2015.7375561.

[4] T. Kawase, M. Okamoto, T. Fukutomi, Y. Takahashi, R. Masuda, and T. Ootake, "Self-Adjustable Speech Enhancement and Recognition System," 2019 IEEE International Conference on Consumer Electronics (ICCE), 2019, pp. 1-2, doi: 10.1109/ICCE.2019.8661925.

[5] B. Widrow and S. D. Stearns, Adaptive signal processing. Prentice-Hall, Inc., 1985.

[6] D. N. Shubhra Dixit, "LMS Adaptive Filters for Noise Cancellation: A Review," International Journal of Electrical and Computer Engineering (IJECE) vol. 7, no. 5, pp. 2520 2529, 2017, doi: 10.11591/ijece.v7i5.pp2520-2529.

[7] T. Ueda and H. Suzuki, "Performance of equalizers employing a re-training RLS algorithm for digital mobile radio communications," 40th IEEE Conf. on Vehicular Tech., 1990, pp. 553-558, doi: 10.1109/VETEC.1990.110383.

[8] S. Kumar, "Directed Searching Optimization-Based Speech Enhancement Technique," Fluctuation and Noise Letters, vol. 19, no. 4, p. 2050035, 2020, doi: 10.1142/S0219477520500352. 
[9] L. B. Asl and M. Geravanchizadeh, "Speech enhancement using sexual reproduction-based PSO," 10th International Conference on Information Science, Signal Processing and their Applications (ISSPA 2010), 2010, pp. 129-132, doi: 10.1109/ISSPA.2010.5605489.

[10] J. Kennedy and R. Eberhart, "Particle swarm optimization," Proceedings of ICNN'95 - International Conference on Neural Networks, 1995, pp. 1942-1948 vol.4, doi: 10.1109/ICNN.1995.488968.

[11] R. Eberhart and J. Kennedy, "A new optimizer using particle swarm theory," MHS'95. Proceedings of the Sixth International Symposium on Micro Machine and Human Science, 1995, pp. 39-43, doi: 10.1109/MHS.1995.494215.

[12] G. Yang, R. Cao, B. Pei, J. Guan, and X. Huang, "Approach of Nonlinear Noise Cancellation based on Neural Network," Electronic Technology \& Information Science, vol. 28, no. 6, pp. 900-902, 2005.

[13] S. Ghalami Osgouei and M. Geravanchizadeh, "Dual-channel speech enhancement based on a hybrid particle swarm optimization algorithm," 2010 5th International Symposium on Telecommunications, 2010, pp. 873-877, doi: 10.1109/ISTEL.2010.5734145.

[14] I. C. Jose and V. Anoop, "Speech Enhancement using LQE and Optimization Techniques," 2020 International Conf. on Comm. and Sig. Proces. (ICCSP), 2020, pp. 751-755, doi: 10.1109/ICCSP48568.2020.9182288.

[15] P. Kunche, G. S. B. Rao, K. V. V. S. Reddy, and R. U. Maheswari, "A new dual channel speech enhancement approach based on accelerated particle swarm optimization (APSO)," International Journal of Intelligent Systems Applications, vol. 6, no. 4, pp. 1-10, 2014, doi: 10.5815/ijisa.2014.04.01.

[16] U. Mahbub, C. Shahnaz, and S. A. Fattah, "An adaptive noise cancellation scheme using particle swarm optimization algorithm," 2010 International Conference on Communication Control and Computing Technologies, 2010, pp. 683-686, doi: 10.1109/ICCCCT.2010.5670753.

[17] L. B. Asl and V. M. Nezhad, "Improved Particle Swarm Optimization for Dual-Channel Speech Enhancement," 2010 International Conference on Signal Acquisition and Processing, 2010, pp. 13-17, doi: 10.1109/ICSAP.2010.30.

[18] L. B. Asl and V. M. Nezhad, "Speech Enhancement Using Particle Swarm Optimization Techniques," 2010 International Conference on Measuring Technology and Mechatronics Automation, 2010, pp. 441-444, doi: 10.1109/ICMTMA.2010.510.

[19] S. Lakshmikanth, K. R. Natraj, and K. R. Rekha, "Performance analysis of industrial noise cancellation with pso based wiener filter using adaptive LMS \& NLMS," 2014 International Conference on Communication and Signal Processing, 2014, pp. 363-368, doi: 10.1109/ICCSP.2014.6949863.

[20] M. K. Ahirwal, A. Kumar, and G. K. Singh, "Adaptive filtering of EEG/ERP through noise cancellers using an improved PSO algorithm," Swarm and Evolutionary Computation, vol. 14, pp. 76-91, 2014, doi: 10.1016/j.swevo.2013.10.001.

[21] K. U. Devi, D. Sarma, and R. Laishram, "Swarm Intelligence based computing techniques in speech enhancement," 2015 International Conference on Green Computing and Internet of Things (ICGCIoT), 2015, pp. 1199-1203, doi: 10.1109/ICGCIoT.2015.7380645.

[22] P. Kunche, G. S. B. Rao, K. V. V. S. Reddy, and R. U. Maheswari, "A new approach to dual channel speech enhancement based on hybrid PSOGSA," International Journal of Speech Technology, vol. 18, no. 1, pp. 45-56, 2015, doi: 10.1007/s10772-014-9245-5.

[23] R. S. Selvi and G. Suresh, "Hybridization of spectral filtering with particle swarm optimization for speech signal enhancement," International Journal of Speech Technology, vol. 19, no. 1, pp. 19-31, 2016, doi: 10.1007/s10772015-9317-1.

[24] R. M. Ramil, S. A. Samad, and A. O. A. Noor, "Objective and subjective evaluations of adaptive noise cancellation systems with selectable algorithms for speech intelligibility," Bulletin of Electrical Engineering Informatics, vol. 7, no. 4 , pp. 570-579, 2018, doi: 10.11591/eei.v7i4.1183.

[25] U. Y. V. Vijayasri Bolisetty and I. Santiprabha, "Speech enhancement using modified wiener filter based MMSE and speech presence probability estimation," International Journal of Informatics and Communication Technology (IJ-ICT), vol. 9, no. 2, pp. 63-72, August 2020, doi: 10.11591/ijict.v9i2.pp63-72.

[26] P. Sun and J. Qin, "Speech enhancement via two-stage dual tree complex wavelet packet transform with a speech presence probability estimator," The Journal of the Acoustical Society of America, vol. 141, no. 2, pp. 808-817, 2017, doi: 10.1121/1.4976049.

[27] P. Kunche, K. V. V. S. Reddy, G. S. B. Rao, and R. U. Maheswari, "A comparative study of BA, APSO, GSA, hybrid PSOGSA and SPSO in dual channel speech enhancement," International Journal of Speech Technology, vol. 18, no. 4, pp. 663-671, 2015, doi: 10.1007/s10772-015-9308-2.

[28] D. Wang, D. Tan, and L. J. S. C. Liu, "Particle swarm optimization algorithm: an overview," Soft Computing, vol. 22, no. 2, pp. 387-408, 2018, doi: 10.1007/s00500-016-2474-6.

[29] T. Lavanya, K. Mrinalini, P. Vijayalakshmi, and T. Nagarajan, "Histogram Matching based Optimized Energy Redistribution for Near End Listening Enhancement," TENCON 2019 - 2019 IEEE Region 10 Conference (TENCON), 2019, pp. 1307-1312, doi: 10.1109/TENCON.2019.8929292.

[30] R. M. Ramli, A. O. A. Noor, and S. A. Samad, "A review of adaptive line enhancers for noise cancellation," Australian Journal of Basic and Applied Sciences, vol. 6, no. 6, pp. 337-352, 2012.

[31] Y. Hu and P. C. Loizou, "Subjective evaluation and comparison of speech enhancement algorithms," Speech Communication, vol. 49, no. 7-8, pp. 588-601, 2007, doi: 10.1016/j.specom.2006.12.006.

[32] NOISEX-92, Aug 1996. [Online]. Available: http://www.speech.cs.cmu.edu/comp.speech/Section1/Data/noisex.html 